\title{
High-Definition Infrared Spectroscopic Imaging
}

\author{
Rohith K. Reddy,b, Michael J. Walsh ${ }^{\mathrm{b}}$, Matthew V. Schulmerich ${ }^{\mathrm{a}, \mathrm{b}}$, P. Scott Carney ${ }^{\mathrm{b}, \mathrm{c}}$, and \\ Rohit Bhargava ${ }^{a, b, c, d,{ }^{*}}$ \\ aDepartment of Bioengineering, University of Illinois at Urbana-Champaign, Urbana, IL \\ bBeckman Institute for Advanced Science and Technology, University of Illinois at Urbana- \\ Champaign, Urbana, IL \\ 'Department of Electrical and Computer Engineering, University of Illinois at Urbana-Champaign, \\ Urbana, IL \\ ${ }^{\mathrm{d}}$ Department of Mechanical Science and Engineering, Micro and Nanotechnology Laboratory and \\ University of Illinois Cancer Center, University of Illinois at Urbana-Champaign, Urbana, IL 61801 \\ USA
}

\begin{abstract}
The quality of images from an infrared (IR) microscope has traditionally been limited by considerations of throughput and signal-to-noise ratio (SNR). An understanding of the achievable quality as a function of instrument parameters, from first principals is needed for improved instrument design. Here, we first present a model for light propagation through an IR spectroscopic imaging system based on scalar wave theory. The model analytically describes the propagation of light along the entire beam path from the source to the detector. The effect of various optical elements and the sample in the microscope is understood in terms of the accessible spatial frequencies by using a Fourier optics approach and simulations are conducted to gain insights into spectroscopic image formation. The optimal pixel size at the sample plane is calculated and shown much smaller than that in current mid-IR microscopy systems. A commercial imaging system is modified, and experimental data are presented to demonstrate the validity of the developed model. Building on this validated theoretical foundation, an optimal sampling configuration is set up. Acquired data were of high spatial quality but, as expected, of poorer SNR. Signal processing approaches were implemented to improve the spectral SNR. The resulting data demonstrated the ability to perform high-definition IR imaging in the laboratory by using minimally-modified commercial instruments.
\end{abstract}

\section{Index Headings}

Mid-infrared; Fourier transform infrared; Spectroscopic imaging; FT-IR imaging; Resolution; Image quality; Modeling; Theory; Scalar wave theory; Microscopy

\section{INTRODUCTION}

Instrumentation for Fourier transform infrared (FT-IR) microspectroscopic imaging typically consists of an interferometer for multiplexed spectral encoding, microscope optics for condensing light and image formation, as well as a focal plane array (FPA) detector for multichannel data recording. ${ }^{1}$ The instrumentation has benefited from nearly 60 years of

(C) 2013 Society for Applied Spectroscopy

*Author to whom correspondence should be sent: rxb@illinois.edu. 
development, with its genesis in point-by-point mapping, ${ }^{2,3}$ and FT-IR microscopy instrumentation that was developed in the $1980 \mathrm{~s}^{4}$. The first instruments contained a singleelement detector that collected all light transmitted by the microscope, while apertures were used to define the spatial resolution. The use of far-field apertures to localize the region illuminated at the focal plane of the microscope implied that the smallest spot size attained was primarily determined by the wavelength of light. Most studies, however, involved larger spot sizes to achieve higher throughput and, consequently, higher signal-to-noise ratio (SNR) in acquired data. Two dogmatic ideas then emerged to dominate IR imaging technology. The first was that significant information could not be derived from areas smaller in dimension than the wavelength of light. While this was indeed true because of a lack of throughput for the point-microscopy case, the elimination of apertures in IR imaging meant that there would still be significant throughput at smaller pixels sizes. The second misconception was that there was no benefit to increasing pixel density beyond the optical diffraction limit imposed by the wavelength, as it would not result in more spatial details being accessible. Evidence to the contrary was available with the use of subwavelength apertures along with the higher throughput of a synchrotron source, making the approach feasible and providing excellent quality data. ${ }^{5}$

In other studies, there was also evidence of improved image quality by subpixel stepping ${ }^{6}$ in mapping experiments, which did not gain much favor on two grounds. The first objection was a practical consideration. Long acquisition time became even longer. The second objection arose due to the lack of an appropriate theory, leading to a mixing of the concepts of the resolution of optical microscopy with the quality of images arising from IR absorption. As emphasized in this manuscript, the two are often used interchangeably while not being equivalent. Though it is correct that a resolution higher than that determined by the optical configuration and wavelength cannot be attained, there is also no denying the improvement in image quality by recording far-field data from areas of dimensions smaller than wavelength. For example, ${ }^{7-9}$ aperture sizes as small as $3 \mu \mathrm{m} \times 3 \mu \mathrm{m}$ provided excellent data when using a synchrotron source at wavelengths much longer than $3 \mu \mathrm{m}$. Spectral quality was retained in this study and maps were sharper. More recent studies ${ }^{10}$ showed improved image quality by combining subpixel images. However, the data themselves have not been collected at the optimal pixel sizes. Coupling an FPA to synchrotron-based microscope systems ${ }^{6,11,12}$ has recently provided stunning improvements in image quality. ${ }^{13}$ Spectra of reasonable quality could be acquired in minutes by using multiple beams, and the multichannel advantages of FPAs allowed for large area coverage. In these advances, the brightness of a synchrotron has been a key factor, and it is unclear if the same benefits are achievable in conventional instruments when using a globar source. While the above are considerable advances in instrumentation, few theoretical analyses and validation of resolution and imaging properties have been undertaken. ${ }^{14,15}$ These analyses, however, did not provide for a rigorous model of the IR microscope and did not examine the case of pixel sampling beyond the conventional wavelength-limited designs. The lack of an appropriate theory to optimize image quality thus remains as a gap in our understanding. This gap is also a barrier to design of tabletop instruments of optimal quality as it is difficult, at present, to make design trade-offs in a quantitative manner.

In this manuscript, we first undertake a theoretical analysis of image formation in IR microspectroscopic imaging. In particular, we focus on determining the best configuration for optimal image quality. We emphasize that attainable resolution and image quality, though related by wavelength and parameters of the optical setup, might not be determined by the same rules. Once the optimal image quality is determined, only then can an examination of the attainable resolution be undertaken. Hence, this is not a detailed discussion of resolution; we focus instead on criteria for obtaining images of the highest possible quality. By using the developed theory, we first determine the pixel size for highest 
image quality permitted by the optical components. While we focus here only on the capabilities of the microscope, there are other theoretical approaches dealing comprehensively with the effects of optical properties of the sample; size and shape of domains; ${ }^{16}$ geometry of acquisition; and microscopy optics on both spectral distortions ${ }^{17,18}$ and image formation. ${ }^{19,20}$ The approach here, therefore, is a more general model that neglects sample detail in the interests of understanding microscope performance. Second, we implement the theoretical recommendations by modifying a commercial system. Finally, we present the integration with previously described noise-reduction strategies to provide improved data quality.

\section{A Model for FT-IR Spectroscopic Imaging}

A theoretical analysis is presented here for the system shown in Fig. 1. Models based on ray (geometric) optics have been especially popular for IR microscopy, but are inadequate for analysis of wavelength-associated effects. Full-scale electromagnetic models, at the other extreme, not only capture all of the physics of image formation including polarization effects, but also involve increased complexity. It is prudent to choose the simplest model that incorporates all the phenomena of interest. Hence, we forgo the full electromagnetic treatment in favor of using the simpler scalar wave theory framework.

Our analysis is based on explicitly calculating the electric field at every plane in the microscope system. To simplify the analysis, we consider a monochromatic component of the field with a wavenumber $v$ and complex amplitude $U .{ }^{1}$ Dependence on $v$ is implied throughout unless otherwise stated. The field can be expressed as a superposition of plane waves described by functions of the form $U(\mathbf{f}) \exp \left\{i 2 \pi\left[\mathbf{f} \cdot \mathbf{r}+f_{z}(\mathbf{f}) z\right]\right\}$, where $\mathbf{f}$ is a twodimensional vector, for instance in Cartesian coordinates, $\mathbf{f}=\left(f_{x}, f_{y}\right)$. The function $f_{z}(\mathbf{f})$ is defined so that $|\mathbf{f}|^{2}+f_{z}^{2}(\mathbf{f})=\bar{v}^{2}$, that is, the plane waves satisfy the Helmholtz equation. For each plane wave constituting the field, its relative contribution, determined by its amplitude, and its relative position, determined by its phase, are both necessary for a complete description of the field. The field and all linear transformations of the field can be represented as a linear combination of these plane waves, which travel at various angles. The term "angular spectrum" is often used ${ }^{21}$ to describe such decomposition. We emphasize that the use of the word "spectrum" in this context should not be confused with the absorption spectrum that is commonly encountered in spectroscopy, and that "spectrum" without the modifier "angular" is meant to be the usual absorption spectrum. Similarly, the term "spatial frequency" refers to the vector $\mathbf{f}$ used above and should not be confused with spectral "frequency." For the convenience of the reader, we have summarized the notation used in the Appendix.

\section{OPTICAL SYSTEM}

The system shown in Fig. 1 can be analyzed by using a modular approach. To that end, we describe each component in the optical system with an operator. This approach provides a convenient means of modifying the analysis to accommodate changes in the instrument. As the system is linear, it is convenient to work in a notation designed for linear algebra, namely the Dirac notation. ${ }^{22}$ Vectors describing the field in a plane at constant axial coordinate $z$ are written $\left|U_{z}\right\rangle$ with Hermitian adjoint $\left\langle U_{z}\right|$. The two-dimensional Fourier component of the field at spatial frequency $\mathbf{f}$ is written $\widetilde{U}(\mathbf{f})=\left\langle\mathbf{f} \mid U_{z}\right\rangle$, and the value of the field at a point $\mathbf{r}$ is given by $U(\mathbf{r})=\left\langle\mathbf{r} \mid U_{z}\right\rangle$. The sample is assumed to be thin, as described by a vector, $|S\rangle$. The detector plane is taken to be at $z_{\mathrm{D}}$. Thus the goal of the analysis is to produce an equation of the form

\footnotetext{
${ }^{1}$ Please consult Table II for a detailed description of the symbols used in the equations throughout this paper.
} 


$$
\left|U_{z_{\mathrm{D}}}\right\rangle=\mathbf{A}|S\rangle
$$

where A describes the optical system.

We first address the operator for propagation through free space between two parallel planes separated by a distance $d$, denoted $\mathbf{K}_{d}$. We assume unidirectional propagation along the axis normal to the planes so that propagation is simply the accumulation of phase. The matrix elements of $\mathbf{K}_{d}$ are given by:

$$
\left\langle\mathbf{f}_{1}\left|\mathbf{K}_{d}\right| \mathbf{f}_{2}\right\rangle=\delta\left(\mathbf{f}_{2}-\mathbf{f}_{1}\right) e^{i 2 \pi f_{z}\left(\mathbf{f}_{1}\right) d}
$$

where $\delta$ is the Dirac delta function, and $\mathbf{f}_{1}$ and $\mathbf{f}_{2}$ denote spatial frequencies. Alternatively, the operator can be constructed as:

$$
\mathbf{K}_{d}=\int \mathrm{d}^{2} f|\mathbf{f}\rangle e^{i 2 \pi f_{z}(\mathbf{f}) d}\langle\mathbf{f}|
$$

It is useful to note that $\mathbf{K}_{d} \mathbf{K}_{d^{\prime}}=\mathbf{K}_{d+d}$.

An interferometer can be thought of as a beam splitter, followed by propagation of two different axial distances. Given arms of length $d_{\mathrm{A}_{1}}$ and $d_{\mathrm{A}_{2}}$, measured from the beam splitter to each of the mirrors, the interferometer is represented by an operator

$$
\mathbf{I}_{d_{\mathrm{A}_{1}, d_{\mathrm{A}_{2}}}}=\frac{1}{2}\left(\mathbf{K}_{2 d_{\mathrm{A}_{1}}}+\mathbf{K}_{2 d_{\mathrm{A}_{2}}}\right)
$$

Next, we consider the effect of a Schwarzschild (also known as a Cassegrain in the literature). Much like a Fresnel lens, the Schwarzschild can be considered to impart onto the field a quadratic phase factor. We represent the Schwarzschild $C$ with an operator $\mathbf{G}_{C}$ :

$$
\mathbf{G}_{C}=\int d^{2} r|\mathbf{r}\rangle Q_{C}(\mathbf{r}) \exp \left[-i \frac{\pi \bar{\nu}}{L_{f}} r^{2}\right]\langle\mathbf{r}|
$$

where $L_{f}$ is the focal length of the Schwarzschild. The quadratic phase factor converts each plane wave incident on the Schwarzschild into a spherical wave converging on its focal plane. The phase relation between plane waves incident on the Schwarzschild along with the imparted quadratic phase determine the final image position and size. $Q_{C}$ is the aperture function (described in detail in the Appendix). It is often the case that the Schwarzschild appears between two propagation steps in the form $\mathbf{K}_{d_{2}} \mathbf{G}_{C} \mathbf{K}_{d_{1}}$. As shown in the Appendix, to a good approximation, when $d_{1}^{-1}+d_{2}^{-1}=L_{f}^{-1}$, the Schwarzschild focuses light from the first plane into the second with a magnification factor $M_{C}=d_{2} / d_{1}$. Thus, it is convenient to define

$$
\mathbf{H}_{C}=\mathbf{K}_{d_{2}} \mathbf{G}_{C} \mathbf{K}_{d_{1}} \approx \int d^{2} f|\mathbf{f}\rangle \mathrm{Q}_{C}(\mathbf{f})\left\langle-M_{C} \mathbf{f}\right|
$$

Clearly, $\mathbf{H}_{C}$ is not uniquely defined unless $d_{1}$ and $d_{2}$ are specified, and care should be taken in observing the context in which it is placed. When the image plane is out-of-focus by a distance $d_{0}$, for example, the propagation operator $\mathbf{K}_{d_{0}}$ can be used to obtain the out-of-focus image using the operator $\mathbf{K}_{d_{0}+d_{2}} \mathbf{G}_{C} \mathbf{K}_{d_{1}}=\mathbf{K}_{d_{0}} \mathbf{H}_{C}$. 
Given a field produced at the source $\left|U_{0}\right\rangle$, at $z=z_{0}$ then propagated to the interferometer at $\mathrm{z}$ $=\mathrm{z}_{\mathrm{I}}$, acted on by the interferometer, then propagated to the first Schwarzschild, $C_{1}$, at $z=$ $z_{C_{1}}$ and then from the Schwarzschild to its focal plane, $z=z_{f_{1}}$, the resultant field is

$$
\left|U_{z_{f_{1}}}\right\rangle=\mathbf{K}_{z_{f_{1}}-z_{C_{1}}} \mathbf{G}_{C_{1}} \mathbf{K}_{z_{C_{1}}-z_{\mathrm{I}}} \mathbf{I}_{d_{\mathrm{A}_{1}}, d} \mathbf{A}_{\mathrm{A}_{2}} \mathbf{K}_{z_{\mathrm{I}}-z_{0}}\left|U_{0}\right\rangle
$$

The sample is modeled as a thin screen. That is, the field on the far side of the sample is assumed to be the product of the sample structure function and the field incident on the sample in the coordinate domain. We formally construct an operator that effects this multiplication. Given a field $|U\rangle$, the operator $\mathbf{U}$ is defined:

$$
U=\int \mathbf{d}^{2} r|r\rangle\langle r \mid U\rangle\left\langle r\left|=\int \mathbf{d}^{2} f \mathbf{d}^{2} f^{\prime}\right| \mathbf{f}\right\rangle \mathbf{f}-\mathbf{f}^{\prime}|U\rangle\left\langle\mathbf{f}^{\prime}\right|
$$

The sample, $|S\rangle$, then interacts with the incident field, $\left|U_{z f_{1}}\right\rangle$, to produce a transmitted field at a plane $z=z_{f_{1}+}$ just past the sample:

$$
\left|U_{z_{f_{1}}+}\right\rangle=\mathrm{U}_{z_{f_{1}}}|S\rangle=\int \mathbf{d}^{2} f \mathbf{d}^{2} f^{\prime}|\mathbf{f}\rangle\left\langle\mathbf{f}-\mathbf{f}^{\prime} \mid U_{z_{f_{1}}+}\right\rangle\left\langle\mathbf{f}^{\prime} \mid S\right\rangle
$$

Substituting from Eq. 7 we find that:

$$
\left|U_{z_{f_{1}}+}\right\rangle=\int \mathbf{d}^{2} f \mathbf{d}^{2} f^{\prime}|f\rangle\left\langle\mathbf{f}-\mathbf{f}^{\prime}\left|\mathbf{K}_{z_{f_{1}}-z_{C_{1}}} \mathbf{G}_{C_{1}} \mathbf{K}_{Z_{C_{1}}-Z_{1}} \mathbf{I}_{d_{\mathrm{A}_{1}}, d_{\mathrm{A}_{2}}} \mathbf{K}_{Z_{1}-Z_{0}}\right| U_{0}\right\rangle \times\left\langle\mathbf{f}^{\prime} \mid S\right\rangle
$$

Note that $\mathbf{I}_{d_{\mathrm{A}_{1}}, d_{\mathrm{A}_{2}}}=\mathbf{K}_{2 d_{\mathrm{A}_{1}}} \mathbf{I}_{0, d_{\mathrm{A}}{ }_{2}-d_{\mathrm{A}_{1}}}$. Assuming no misalignment, i.e., when the system is in focus, the propagation operators can be absorbed into the magnification factor of $\mathbf{H}_{C_{1}}$. Thus,

$$
\left|U_{z_{f_{1}}+}\right\rangle=\int \mathrm{d}^{2} f \mathrm{~d}^{2} f^{\prime}|f\rangle\left\langle\mathrm{f}-\mathrm{f}^{\prime}\left|\mathrm{H}_{C_{1}} \mathrm{I}_{0, d_{\mathrm{A}_{2}}-d_{\mathrm{A}_{1}}}\right| U_{0}\right\rangle\left\langle\mathrm{f}^{\prime} \mid S\right\rangle
$$

where $\mathbf{H}_{C_{1}}=\mathbf{K}_{z_{f_{1}}-z C_{1}} \mathbf{G}_{C_{1}} \mathbf{K}_{z_{C_{1}}-z_{0}+2 d_{\mathrm{A}_{1}}}$. Evaluating Eq. 11 explicitly, we find that the field just past the sample is given by:

$$
\left|U_{z_{f_{1}}+}\right\rangle=\frac{1}{2} \int d^{2} f d^{2} f^{\prime}|\mathbf{f}\rangle Q_{C_{1}}\left(\mathbf{f}^{\prime} / M_{C_{1}}\right) \times\left\{1+\exp \left[i 2 \pi f_{z}\left(\mathbf{f}^{\prime}\right)\left(2 d_{\mathrm{A}_{2}}-2 d_{\mathrm{A}_{1}}\right)\right]\right\} \times\left\langle\mathbf{f}^{\prime} \mid U_{0}\right\rangle\left\langle\mathbf{f}+\mathbf{f}^{\prime} / M_{C_{1}} \mid S\right\rangle
$$

where it can be noted that $\langle\mathbf{f} \mid S\rangle$ is the Fourier component of the sample at spatial frequency $\mathbf{f}$. In the case that the source consists of a point source far from the first Schwarzschild, $\left\langle\mathbf{f} \mid U_{0}\right\rangle$ $\approx A_{0}$, where $A_{0}$ is a constant and inclination factors of the form $1 / f_{z}$ have been neglected. Then the field is given by:

$$
\left|U_{z_{f_{1}}+}\right\rangle=\frac{A_{0}}{2} \int d^{2} f d^{2} f^{\prime}|\mathbf{f}\rangle Q_{C_{1}}\left(\mathbf{f}^{\prime} / M_{C_{1}}\right) \times\left\{1+\exp \left[i 2 \pi f_{z}(\mathbf{f})\left(2 d_{\mathrm{A}_{2}}-2 d_{\mathrm{A}_{1}}\right)\right]\right\}\left\langle\mathbf{f}+\mathbf{f}^{\prime} / M_{C_{1}} \mid S\right\rangle
$$

Light propagation from the Schwarzschild $C_{1}$ to the sample and to the Schwarzschild $C_{2}$ are illustrated in Fig. 2.

The propagation of the field after the sample, $\left|U_{z f_{1}+}\right\rangle$, to the field at the detector, $\left|U_{z_{D}}\right\rangle$, takes place through two more Schwarzschilds. The Schwarzschild $C_{2}$, located effectively at 
$z=z_{C_{2}}$, focuses the sample plane to a plane $z=z_{f_{2}}$ such that $1 /\left(z_{f_{1}}-z_{C_{2}}\right)+1 /\left(z_{C_{2}}-z_{f_{2}}\right)=1 / L_{f_{2}}$, where $L_{f_{2}}$ is the focal length of Schwarzschild $C_{2}$. Similarly, the Schwarzschild $C_{\mathrm{D}}$ located at $\mathrm{z}=\mathrm{z}_{C_{\mathrm{D}}}$, focuses light from the plane $z=\mathrm{z}_{f_{\mathrm{D}}}$ onto the detector plane at $z=z_{\mathrm{D}}$ such that $1 /$ $\left(z_{C_{\mathrm{D}}}-z_{f_{\mathrm{D}}}\right)+1 /\left(Z_{\mathrm{D}}-z_{C_{\mathrm{D}}}\right)=1 / L_{f_{\mathrm{D}}}$, where $L_{f_{\mathrm{D}}}$ is the focal length of Schwarzschild $C_{\mathrm{D}}$. Misalignment of the focal planes such that $z_{f_{\mathrm{D}}} \neq z_{f_{2}}$ can be taken into account with the propagator $\mathbf{K}_{z f_{\mathrm{D}}-z f_{2}}$. Thus,

$$
\begin{gathered}
\left|U_{z_{d}}\right\rangle=\mathbf{H}_{C_{D}} \mathbf{K}_{z_{f_{D}}-z_{f_{2}}} \mathbf{H}_{C_{2}}\left|U_{z_{f_{1}}+}\right\rangle \\
=\int \mathrm{d}^{2} f \mathrm{~d}^{2} f^{\prime} \mathrm{H}_{C_{\mathrm{D}}} \mathrm{K}_{Z_{f_{\mathrm{D}}}-Z_{f_{2}}} \mathrm{H}_{C_{2}}|\mathrm{f}\rangle\left\langle\mathrm{f}-\mathrm{f}^{\prime}\left|H_{C_{1}} I_{0, d_{A_{2}}-d_{A_{1}}}\right| U_{0}\right\rangle \times\left\langle\mathrm{f}^{\prime} \mid S\right\rangle
\end{gathered}
$$

This expression is now straightforward to evaluate. We assume that $z_{f \mathrm{D}}=z_{f_{2}}$ (the focal planes are aligned). We find explicitly that:

$$
\begin{aligned}
\left|U_{z_{\mathrm{D}}}\right\rangle & =\frac{1}{2} \int d^{2} f d^{2} f^{\prime}|\mathbf{f}\rangle Q_{C_{\mathrm{D}}}(f) Q_{C_{2}}\left(-M_{C_{\mathrm{D}}} \mathbf{f}\right) Q_{C_{1}}( \\
& \left.-\mathbf{f}^{\prime} / M_{C_{1}}\right) \\
& \times\left\{1+\exp \left[i 2 \pi f_{z}\left(\mathbf{f}^{\prime}\right)\left(2 d_{\mathrm{A}_{2}}-2 d_{\mathrm{A}_{1}}\right)\right]\right\}\left\langle\mathbf{f}^{\prime} \mid U_{0}\right\rangle \times\left\langle\mathbf{f}^{\prime} / M_{C_{1}}\right. \\
& +M_{C_{2}} M_{C_{\mathrm{D}}} \mathbf{f}|S\rangle
\end{aligned}
$$

Define $M=M_{C_{D}} M_{C_{2}}$ and compute

$$
\left\langle\mathbf{f} \mid U_{z_{\mathrm{D}}}\right\rangle=\frac{1}{2} Q_{C_{\mathrm{D}}}(\mathbf{f}) Q_{C_{2}}\left(-M_{C_{\mathrm{D}}} \mathbf{f}\right) \int d^{2} f^{\prime} Q_{C_{1}}\left(-\mathbf{f}^{\prime} / M_{C_{1}}\right) \times\left\{1+\exp \left[i 2 \pi f_{z}\left(\mathbf{f}^{\prime}\right)\left(2 d_{\mathrm{A}_{2}}-2 d_{\mathrm{A}_{1}}\right)\right]\right\} \times\left\langle\mathbf{f}^{\prime} \mid U_{0}\right\rangle\left\langle\mathbf{f}^{\prime} / M_{C_{1}}+M \mathbf{f} \mid S\right\rangle
$$

The above expression is true for any spatially heterogeneous sample and has no restriction on its constituent spatial frequencies. Note that $M$ is typically larger than 1 (i.e., the detector elements are bigger than the corresponding areas on the sample).

In order to illustrate the significance of Eq. 16, consider a case where the sample is a point object. In this case, $\left\langle\mathbf{f}^{\prime} / M_{C_{1}}+M \mathbf{f} \mid S\right\rangle=1$, giving

$$
\begin{gathered}
\left\langle\mathbf{f} \mid U_{z_{\mathrm{D}}}\right\rangle=\frac{1}{2} Q_{C_{\mathrm{D}}}(\mathbf{f}) Q_{C_{2}}\left(-M_{C_{\mathrm{D}}} \mathbf{f}\right) \int d^{2} f^{\prime} Q_{C_{1}}\left(-\mathbf{f}^{\prime} / M_{C_{1}}\right) \times\left\{1+\exp \left[i 2 \pi f_{z}\left(\mathbf{f}^{\prime}\right)\left(2 d_{\mathrm{A}_{2}}-2 d_{\mathrm{A}_{1}}\right)\right]\right\}\left\langle\mathbf{f}^{\prime} \mid U_{0}\right\rangle \\
=\frac{1}{2} Q_{C_{\mathrm{D}}}(\mathbf{f}) Q_{C_{2}}\left(-M_{C_{\mathrm{D}}} \mathbf{f}\right) B_{0}
\end{gathered}
$$

Here, $B_{0}$, the integral from Eq. 17 , is independent of $\mathbf{f}$ and physically denotes the field at the sample plane for a point object. The corresponding intensity at the detector plane is

$$
\left\langle U_{z_{\mathrm{D}}} \mid \mathbf{f}\right\rangle\left\langle\mathbf{f} \mid U_{z_{\mathrm{D}}}\right\rangle-\frac{B_{0}^{2}}{4}\left[Q_{C_{\mathrm{D}}}(\mathbf{f}) Q_{C_{2}}\left(-M_{C_{\mathrm{D}}} \mathbf{f}\right)\right] *\left[Q_{C_{\mathrm{D}}}(\mathbf{f}) Q_{C_{2}}\left(-M_{C_{\mathrm{D}}} \mathbf{f}\right)\right]
$$

where * represents convolution.

In a case where the source is completely incoherent, i.e., $\left\langle\left\langle U_{0} \mid \mathbf{r}^{\prime}\right\rangle\left\langle\mathbf{r} \mid U_{0}\right\rangle\right\rangle_{\text {en }}=I_{0}(\mathbf{r}) \delta\left(\mathbf{r}-\mathbf{r}^{\prime}\right)$, the intensity at the detector plane is 


$$
\left\langle\left\langle U_{z_{\mathrm{D}}} \mid \mathbf{r}^{\prime}\right\rangle\left\langle\mathbf{r} \mid U_{z_{\mathrm{D}}}\right\rangle\right\rangle_{e n}=\int d^{2} r I_{0}\left(\mathbf{r}^{\prime}\right)\left|h\left(\mathbf{r} ; \mathbf{r}^{\prime}\right)\right|^{2}
$$

Where $h\left(\mathbf{r} ; \mathbf{r}^{\prime}\right)$ is the transfer function from the coherent case and $I_{0}(\mathbf{r})$ is the source intensity. The subscript $e n$ above denotes an ensemble average over constituent random processes. By using the equations presented in this section, we proceed to compute the spatial sampling rate and pixel size required to record all the information available from the FT-IR spectroscopic imaging system.

\section{Pixel Size}

An analysis of the equations for the FT-IR imaging system provides insight into optical design and image formation. The explicit form of $Q_{C_{i}}$ in terms of NA is derived in the Appendix (Eq. A29, Eq. A30, and Eq. A31). It can be observed from Eq.16 that the spatial frequencies on the detector are always less than $\min \left(v \mathrm{NA}_{C_{2} \text { out }} / M, v \mathrm{NA}_{C_{\text {Dout }}}\right)$ because the support of the data is the intersection of the support of $Q_{C_{2}}\left(-M_{C_{\mathrm{D}}} \mathbf{f}\right)$ and $Q_{C_{\mathrm{D}}}(\mathbf{f})$. When the pre-optics to the detector are well designed, then $v \mathrm{NA}_{C_{2} \text { out }} / M, \unlhd v \mathrm{NA}_{C_{D} \text { out }}$, providing the limiting field on the detector to be a spatial frequency of $v \mathrm{NA}_{C_{2} \text { out }} / M$. As the detector records intensity and not fields, ${ }^{21}$ the intensity $I(\mathbf{r})=U^{*}(\mathbf{r}) U(\mathbf{r})$ has a spatial frequency bound of $2 \times v \mathrm{NA}_{C_{2} \text { out }} / M$. We emphasize that in order to faithfully record the entire intensity image without any loss of information, the spatial sampling rate according to the Nyquist criterion ${ }^{21}$ has to be at least twice this limiting frequency, or $4 v \overline{\mathrm{NA}}_{C_{2} \text { out }} / M$. The pixel size is inversely related to the sampling rate, i.e., $L_{\text {pixel }}=M / 4 v \overline{\mathrm{NA}}_{C_{2} \text { out }}$. The wavenumber, $v$, in mid-IR spectroscopic imaging typically varies between 600 and 4000 $\mathrm{cm}^{-1}\left(0.4 \mu \mathrm{m}^{-1}\right)$. Therefore, when using Eq. 16, the equivalent pixel size on the sample has to be $1 /\left(4 \times 0.4 \mathrm{NA}_{C_{2} \text { out }}\right) \mu \mathrm{m}$. Thus, for $\mathrm{NA}_{C_{2} \text { out }}=0.5$, the effective pixel size on the sample is $125 \mu \mathrm{m}$ and for $\mathrm{NA}_{C_{2} \text { out }}=0.65$, the effective pixel size is $0.96 \mu \mathrm{m}$. It must be emphasized that the value of pixel size calculated above is not the same as resolution. It is in fact smaller than the resolution as defined by the Rayleigh criterion. ${ }^{21}$ However, it reflects the least sampling rate required to utilize all the information passed by the system in FT-IR spectroscopic imaging.

While we have focused on image quality, a number of other insights are also apparent. In some detectors, for example, it can be advantageous to have a concave mirror (instead of a Schwarzschild) that can be easily be incorporated into the model by making the term corresponding to the central obscuration i.e., an inner numerical aperture of zero $\mathrm{NA}_{C_{\mathrm{D}}}^{C_{\mathrm{D}} \text { out }}$ in The absence of a central obscuration increases light throughput significantly. We suggest that the Schwarzschild used in commercial instruments be replaced with this mirror. This modification will result both in better image quality and lower instrument costs. Although the above derivation uses a transmission mode measurement, the same analysis can be performed for transflection mode. In the case of transflection-absorption measurement when using a beam-splitter and full Schwarzschild illumination, Schwarzschilds $C_{1}$ and $C_{2}$ correspond to the same Schwarzschild, and light travels through the sample twice, once before reflection and once after. Hence the sampling rates and pixel sizes are the same in both cases.

\section{Experiments and Simulations}

Instrumentation-A Varian 7000 spectrometer coupled to a UMA-400 microscope was used to perform two sets of experiments with parameters listed in Table I. System 1 uses accessories standard to the Varian microscope. System 2 uses an Edmund Optics (NA = 0.65, 74× magnification) Schwarzschild for $C_{2}$. The two systems make comparisons easy, because parameters other than Schwarzschild $C_{2}$ are the same. The only difference is in the 
effective pixel size on the sample and $\mathrm{NA}_{C_{2} \text { out }}$. The detector's pixel size is $40 \mu \mathrm{m}$. The interferometer is operated in the step-scan mode at a stepping rate of $200 \mathrm{~Hz}$. Data were acquired at every other zero crossing (a undersampling ratio of 2) of an $\mathrm{He}-\mathrm{Ne}$ laser for a free-scanning spectral range of $7900-0 \mathrm{~cm}^{-1}$. Time to acquire a $128 \times 128$ pixel data set by using system 2 was $25 \mathrm{~s}$ for eight co-additions. For the full format of the detector $(128 \times 128$ pixels), the fastest acquisition of an $8 \mathrm{~cm}^{-1}$ resolution data set (undersampling ratio of 4) is less than $1 \mathrm{~s}$ and is limited by FPA speed $(1.6 \mathrm{kHz})$. A Fourier transform of the recorded data was carried out with the Norton-Beer medium apodization function. Data were truncated and stored as absorbance after a ratio against an appropriate background data set.

Data extraction and image processing were performed with a hyperspectral imaging software package, ENvironment for Visualizing Images (ENVI). In ENVI, the twodimensional Fourier transform routines are in built. Modules for further data processing were written in-house using IDL 7.1 and MATLAB 7. The two imaging systems, with parameters shown in Table I (parameters 1 through 6) are simulated. The simulations were performed on a quad core computer with a NVIDIA GeForce GTX 580 GPU and 12 GB of RAM. Modules and functions for the simulations were written in house by using MATLAB 7. The simulations compute the detector field and intensity for incoherent illumination by using the system parameters (Table I, parameters 1 through 6) and object as inputs. Software is implemented based on the theory and algorithms described here. Simple structures can be simulated in minutes, while entire data sets for morphologically complex samples can require days of computational time on a desktop computer. The run time for simulations is about $20 \mathrm{~s}$ for each wavenumber for the samples described here.

Prostate and breast tissue microarrays (TMAs) were used as a platform for high-throughput sampling. ${ }^{23}$ TMAs consist of a large number of small $(\approx 1 \mathrm{~mm}$ in diameter) tissue sections arranged in a grid pattern on a substrate. This technique facilitates and streamlines acquisition of data from several patients and provides diversity to the sample population under observation. Breast and prostate tissue microarrays were obtained from the University of Illinois-Chicago (kindly provided by Dr. Andre Kajdacsy-Balla) and from US Biomax (no. BR1003). One $5 \mu \mathrm{m}$-thick tissue section from each array was placed on a $B a F_{2}$ substrate for IR imaging and serial $5 \mu \mathrm{m}$-thick sections were placed on standard glass slides for hematoxylin and eosin staining. IR imaging data were acquired from a normal breast tissue core that contained a region comprising terminal ductal lobular units, as well as from a normal prostate tissue core from the region of a small blood vessel. A standard chrome-onglass USAF 1951 target (Edmund Optics) was used as a test sample to test both spectral and spatial performance. The thickness of chrome is of the order of $(100 \mathrm{~nm})$ and about $10 \%$ of incident light is transmitted through the chrome (experimentally measured).

\section{RESULTS AND DISCUSSION}

Our first step in analyzing the performance of the imaging systems was to validate simulations with recorded data by using a gold standard for comparison. As common in other forms of microscopy, a standard USAF 1951 target, consisting of chrome on glass, was chosen. The chrome provides a large attenuation (absorbance) with negligible thickness. It also provides high contrast for visible light microscopy. Hence, it is an ideal sample for simulation and validation. We first recorded visible-light images for a standard USAF microscope target. The recorded image was binarized to remove any edge blurring or grayscale values in the simulation. An absorbance of unity was assigned to the chrome region, and the resulting image was used for simulation. Regions without the chrome were assigned a transmittance of unity (zero absorbance). Simulations were carried out for both the instrument configurations described previously. The light source is assumed to be spatially incoherent, as in Eq. 20. Spectroscopic imaging data were also recorded with the 
target. We analyzed groups 6 and 7, and elements 1 through 6 , as these represent the highest image quality typically encountered in IR microscopy. Images of the standard sample employed for simulation, absorbance images from simulated data, and absorbance images from the recorded data corresponding to the two instrument configurations are shown in Fig. 3. In order to facilitate comparison of simulations, we used the same pixel size for both optical configurations (although different NA lenses are often associated with different magnifications). The chosen pixel size for simulations of $0.36 \mu \mathrm{m}$ will not be a limiting factor in the resulting image quality. The USAF target is designed to enable facile calculation of the frequency response of the imaging systems as well as to illustrate resolution capabilities via images. In simulations, we emphasize again that the sample is assumed to be infinitesimally thin to avoid effects associated with thickness. Comparing the two simulated absorbance images (Figs. $3 b$ and $3 f$ ) with the same pixel size shows the effects of increasing NA. As expected, an increase in the NA provides for higher quality and higher resolution images. Nevertheless, the enhancement is not especially striking. A more obvious degradation in image quality is evident when the pixel size increased from 1.1 (Fig. 3f) to $5.5 \mu \mathrm{m}$ (Fig. 3g), while all other parameters were constant. This dramatic change in image quality emphasizes the need for carefully choosing the pixel size. The combined effects of choosing a higher-NA lens and the appropriate sampling (small pixel size) can result in significantly improved image quality (cf. Figs. $3 \mathrm{~b}$ and $3 \mathrm{~g}$ ). To experimentally validate these predicted improvements in image quality, we set up two optical configurations described in Table I on the same commercial microscope and obtained data on the same USAF 1951 target used in simulations. Our goal was to minimally modify (by changing one lens) the existing commercial system. Hence, we did not alter the condenser. Matching the throughput via the image formation lens and condenser is likely to result in better SNR, but will not materially change the appearance of the images, as the image quality depends only on the angular spectral bandwidth of the image formation lens. Experimental results for Fig. $3 \mathrm{c}, \mathrm{NA}=0.65$, pixel size $=1.115 \mu \mathrm{m}$, and Fig. $3 \mathrm{~h}, \mathrm{NA}=0.50$, pixel size $=5.5 \mu \mathrm{m}$, are presented for comparison. A dramatic improvement in image quality is observed experimentally for the smaller pixel size. Expanding a smaller region for comparison in Figs. $3 \mathrm{~d}$ and $3 \mathrm{e}$, and $3 \mathrm{i}$ and $3 \mathrm{j}$, the three smaller bars are distinguishable only in the higher NA, smaller pixel-size setup. This qualitatively demonstrates the gain in image quality and resolution when using a higher-NA system along with the appropriate pixel spacing. In all cases, as expected, we also note that the recorded data are of somewhat lower quality than the simulations. This is a result of edge effects in the sample, finite detector sizes, imperfect optics and experimental noise in the recorded data.

While the images from simulation and recorded data agree well, they do not provide a quantitative understanding of the performance improvement and limits of the two configurations. Therefore, we quantitatively analyzed the performance of the systems as a function of the spatial frequency. The frequency response of the optical system is often characterized by the modulation or contrast transfer function of the system. While optical systems typically employ intensity values to examine the imaging system performance, here we use the spatial frequencies in Fig. 3a and the corresponding absorbance values from simulations and recorded data to plot the absorbance contrast. The absorption contrast ratio (ACR) is defined as the difference in maximum and minimum absorbance observed at a specific spatial frequency compared with that observed in the ideal case. The ideal difference between high and low absorbance is obtained by comparing absorbance values from regions on the sample with no absorbance (for example, the region used to collect a background spectrum) and a relatively homogeneous region of high absorbance (for example, on the large square in a USAF 1951 target). Figure 4a shows a plot of the absorbance contrast ratio as a function of spatial frequency at $3950 \mathrm{~cm}^{-1}$. As expected, ACR is highest at low spatial frequencies and decreases with increasing spatial frequency until zero (no measurable contrast). The absorption contrast ratio for the lower-NA system 
reaches zero at a lower spatial frequency than that of the high NA one as is evident from the polynomial fit (solid lines in the figure) to simulation data. It can be seen in the experimental data that the high-NA system resolves all bars including the smallest bars (group no. 7) available on the USAF 1951 target. The frequency at which ACR reaches zero can be predicted via simulations to be about $0.4 \mu \mathrm{m}^{-1}$ ( $\cong 400$ line pairs per $\mathrm{mm}$ ). Contrast decreases with reduction in pixel size, because light intensity per pixel reduces. As a result, the increased imaging capability is partially offset by a decreased spectral SNR in the resulting absorbance images. While predictions from simulations agree well with measured ACR for the high-NA system, there is some disagreement for the lower-NA setup at high spatial frequencies. This difference is observed at approximately $0.08 \mu \mathrm{m}^{-1}(\approx 80$ line pairs per $\mathrm{mm})$. Experimentally, the lower-NA system cannot resolve bars beyond a spatial frequency of $0.115 \mu \mathrm{m}^{-1}(\approx 115$ line pairs per $\mathrm{mm}$ ). This is lower than the theoretically predicted 0.15 $\mu \mathrm{m}^{-1}$ ( $\approx 150$ line pairs per $\mathrm{mm}$ ). This can be attributed to the finite pixel size preventing an accurate recording of the data. This comparison between ACR of the high- and low-NA systems, enabled by the developed theoretical understanding, provides convincing proof that the designs of present-day instrumentation are not permitting optics-limited performance and can be rapidly and easily reconfigured to provide significantly higher imaging performance.

Though the previous analysis provides the smallest observable features, the relationship of these features to the optimal pixel size needs to be quantified. We examined the pixel size at the sample plane required for optimally sampling the signal as a function of NA and wavelength (Fig. 4b). While the optimal pixel size can also be calculated analytically, this figure provides the design parameters for instruments should a practitioner develop an instrument for high performance at any specific wavelength while using a specific lens. As expected, the recommended pixel size decreases with decreasing design wavelength. The dependence on NA is rather striking as well. It is noteworthy that most commercially available systems provide reasonably high NA, but the pixel size is not commensurate with the optical components. Thus, an improvement in incorporating a higher-NA lens is not likely to be as dramatic as using the optimal pixel size in current commercial systems. We note that this plot is based on the maximum attainable spatial frequencies for thin objects, and larger sample thicknesses are likely to achieve poorer contrast for the limiting spatial frequency.

The pixel sizes recommended will provide the highest image quality provided by the microscope, unless limited by the sample. The optimal pixel size and the resolution of an imaging system are also related. According to the Rayleigh criterion, for example, the attainable resolution is $0.61 /(v \overline{N A})$. We propose to sample at $0.25 /(v \mathrm{NA})$ in order to convert the analog signal into a digital readout without information loss. We note that the pixel size proposed is significantly smaller than the resolution. This result is contrary to prevailing wisdom in that it is widely believed that no further improvement is possible once a pixel size equal to the resolution has been attained. Indeed, mixing the concepts of resolution and pixel sizes for correct signal sampling has led to significant confusion in IR microscopy. We emphasize that our suggestions for improved data quality are not a contravention of the resolution criterion. Consider the case of pixel spacing resulting in optimal resolution. To resolve two objects, we need more than two measurements when the data are digitized. For example, consider two point objects that are centered on a pixel each. To entirely resolve these objects, appropriate sampling implies that there be a pixel on either side of the object to separate them from each other and any other neighboring objects. Therefore, at least five pixels are needed to resolve two point objects. A more formal discussion of sampling for optical microscopy ${ }^{6,24}$ and its extension to IR microscopy using this signal processing approach is provided elsewhere. Our approach is distinct from these methods and incorporates light transmission though an entire imaging system and provides calculations 
based on absorbance. We note that the resolution of an instrument cannot be correctly evaluated unless the pixel size is appropriate (smaller). Even if two objects cannot be resolved into their appropriate shapes by using smaller pixels, the detail in higher pixeldensity images is higher. Two point objects, for example, will appear as dumbbells or ovals. Hence, even for systems or wavelengths in which the pixel size is smaller than required for correctly sampling the spatial frequencies permitted by the optics, an improvement in image quality might be observed. For a detailed discussion, we refer the reader to the Appendix.

Data from the two optical configurations described in Table I are shown in Fig. 5. Data are recorded on prostate tissue that is prepared with methods previously reported, ${ }^{25-27}$ and images are obtained with the absorbance of the asymmetric $\mathrm{C}-\mathrm{H}$ stretching vibrational mode (at $2962 \mathrm{~cm}^{-1}$ ). The top row demonstrates the improvement in image quality attained with the higher-NA lens. The bottom row presents the spatial frequency content of corresponding images. It can be observed from Figs. 5e-5h that there are relatively higher spatial frequencies present in the data acquired with the higher-NA lens, signifying that there is degradation of image quality, i.e., information loss in increasing the pixel size from 1.115 to $5.5 \mu \mathrm{m}$. There is a noticeable set of high spatial frequencies present in the data acquired at the higher NA at the shorter wavelength. Even for longer wavelengths, the information content difference is relatively small, but not zero. To capture the highest image quality across the spectrum, hence, the pixel size should be calculated based on the highest wavenumber measured in the experiment. This statement is both rigorously and intuitively correct. From a practical perspective, however, a small pixel size calculated at the highest wavenumber also reduces the throughput significantly across the rest of the spectrum. To address this trade-off in an optimal manner, we recommend that the highest wavenumber at which pixel size should be calculated should depend on the typical experiments to be performed by the imaging system. This small adjustment from the correct sampling at the highest recorded wavenumber to that at the highest usable wavenumber for image generation leads to the concept of an optimal pixel size. For most studies in the mid-IR, the 4000 to $400 \mathrm{~cm}^{-1}$ spectral region is most interesting. For He-Ne laser reference-based systems, an undersampling of the reference signal by a factor of four typically implies that the allowable free-scanning spectral range is $3950-0 \mathrm{~cm}^{-1}$. An optimal pixel size at the high end of this range is $0.974 \mu \mathrm{m}$. In most experiments, especially with biological systems, vibrational modes at this high limit are very rarely encountered. A more practical high wavenumber region is $3400 \mathrm{~cm}^{-1}$, which is in the vicinity of the absorption peak of $\mathrm{O}-\mathrm{H}$ and $\mathrm{N}-\mathrm{H}$ stretching-associated vibrational modes. Therefore, we calculate the optimal pixel size at this wavenumber and the predicted pixel size is $1.13 \mu \mathrm{m}$ on a side. As we sought to implement the concept of optimal pixel sampling on a commercial imaging system without extensive hardware modifications, the measured pixel size of $1.115 \mu \mathrm{m}$ can be deemed acceptably close. Our suggested optimal pixel size is approximately fourfold larger than a similar setup when using the synchrotron. ${ }^{13}$ It is notable that intensity considerations are secondary for a synchrotron source-based system because of the exceptional flux and a pixel size of $0.54 \mu \mathrm{m}$ on a side was used. Relaxing the very strict condition with a more practical calculation here, we maximize the spectral quality when using a globar source, without compromising the image quality in any appreciable manner. The image quality presented here is likely of the highest quality that will be observed in commonly analyzed biomedical material or forensic samples regardless of the source.

Though image quality is improved with a smaller pixel size, there is a corresponding decrease in throughput if the same source and fore-optics are employed. The approximate 25 -fold reduction in pixel area between the two configurations here implies that acquisition time would need to be increased 625-fold (other factors being constant) if the data quality is to be recovered by signal averaging. ${ }^{28,29}$ Some of the loss is mitigated by increased throughput, as proposed when using a synchrotron, ${ }^{13}$ or by increased integration time of the 
FPA, as in the experiments conducted here. We observed a six- to eightfold decrease in recorded signal when using the higher-NA lens compared with the lower-NA system. Hence, the need to signal average is not especially drastic. Further, there are other methods to increase SNR. We previously proposed computational noise reduction ${ }^{30,31}$ to obtain a significant gain in SNR without the corresponding increase in data collection time. The utility of this idea is presented in Fig. 6. A significant increase in spectral SNR (Fig. 6c) without observable loss in image quality (Figs. 6a and 6b) can be observed. Thus, a tabletop, high-definition IR imaging system is indeed feasible and can provide both excellent spectra and spatial image quality.

\section{CONCLUSION}

A complete theoretical understanding of the image formation in an IR microscope was provided by using a rigorous theoretical model. The model was used to predict the optimal pixel size at the sample plane that would provide the highest image quality. Simulations demonstrated that the effects of the higher-NA systems arose from an increased acceptance of angular frequencies and resulted in higher-resolution images, whereas optimal pixel sampling demonstrated dramatic improvements in image detail for a specific NA. The results of simulations were validated with measurements on two different configurations. A tabletop, high-definition FT-IR spectroscopic imaging system was demonstrated by minimally modifying a commercial system. The resulting data when using this highdefinition system can be of high spatial and spectral quality by using conventional signal averaging and/or emerging signal processing methods. The development of a theoretical understanding and its application to microscope design and acquisition of high-definition data should spur improved applications in many fields where IR imaging is applied. ${ }^{33}$

\section{Acknowledgments}

The project described was supported by awards R01CA138882 and R01EB009745 from the U.S. National Institutes of Health. M.V. Schulmerich acknowledges support through Congressionally Directed Medical Research Program Postdoctoral Fellowship BC101112.

\section{References}

1. Lewis EN, Treado PJ, Reeder RC, Story GM, Dowrey AE, Marcott C, Levin IW. Fourier Transform Spectroscopic Imaging Using an Infrared Focal-Plane Array Detector. Anal Chem. 1995; 67(19): 3377-3381. [PubMed: 8686889]

2. Barer R, Cole ARH, Thompson HW. Infra-red Spectroscopy with the Reflecting Microscope in Physics, Chemistry and Biology. Nature. 1949; 163(4136):198-201. [PubMed: 18109152]

3. Gore RC. Infrared Spectrometry of Small Samples with the Reflecting Microscope. Science. 1949; 110(2870):710-711. [PubMed: 15397441]

4. Kwiatkoski JM, Reffner JA. FT-IR Microspectrometry Advances. Nature. 1987; 328:837-838.

5. Jamin N, Dumas P, Moncuit J, Fridman WH, Teillaud JL, Carr GL, Williams GP. Highly Resolved Chemical Imaging of Living Cells by Using Synchrotron Infrared Microspectrometry. P Natl Acad Sci USA. 1998; 95(9):4837-4840.

6. Carr GL. Resolution Limits for Infrared Microspectroscopy Explored with Synchrotron Radiation. Rev Sci Instrum. 2001; 72:1613-1619.

7. Dumas P, Jamin N, Teillaud JL, Miller LM, Beccard B. Imaging Capabilities of Synchrotron Infrared Microspectroscopy. Faraday Discuss. 2004; 126:289-302. [PubMed: 14992414]

8. Briki F, Busson B, Kreplak L, Dumas P, Doucet J. Exploring a Biological Tissue from Atomic to Macroscopic Scale Using Synchrotron Radiation: Example of Hair. Cell Mol Biol (Noisy-le-Grand, France). 2000; 46(5):1005-1016.

9. Miller LM, Dumas P. Chemical Imaging of Biological Tissue with Synchrotron Infrared Light. Biochim Biophys Acta. 2006; 1758(7):846-857. [PubMed: 16781664] 
10. Offroy M, Roggo Y, Milanfar P, Duponchel L. Infrared Chemical Imaging: Spatial Resolution Evaluation and Super-Resolution Concept. Anal Chim Acta. 2010; 674(2):220-226. [PubMed: 20678633]

11. Bhargava, R.; Levin, I. Spectrochemical Analysis Using Infrared Multichannel Detectors. New York: Wiley-Blackwell; 2005.

12. Petibois C, Cestelli-Guidi M, Piccinini M, Moenner M, Marcelli A. Synchrotron radiation FTIR imaging in minutes: A First Step Towards Real-Time Cell Imaging. Anal Bioanal Chem. 2010; 397(6):2123-2129. [PubMed: 20517599]

13. Nasse MJ, Walsh MJ, Mattson EC, Reininger R, Kajdacsy-Balla A, Macias V, Bhargava R, Hirschmugl CJ. High-Resolution Fourier-Transform Infrared Chemical Imaging with Multiple Synchrotron Beams. Nature Methods. 2011; 8(5):413-416. [PubMed: 21423192]

14. Lasch P, Naumann D. Spatial Resolution in Infrared Microspectroscopic Imaging Of Tissues. Biochimica et Biophysica Acta. 2006; 1758(7):814-829. [PubMed: 16875659]

15. Widengren, J.; Mets, U. Single Molecule Detection in Solution. Wiley Online Library; 2002.

16. Davis BJ, Carney PS, Bhargava R. Theory of Infrared Microspectroscopy for Intact Fibers. Anal Chem. 2011; 83(2):525-532. [PubMed: 21158469]

17. Davis BJ, Carney PS, Bhargava R. Theory of Midinfrared Absorption Microspectroscopy: I. Homogeneous Samples. Anal Chem. 2010; 82(9):3474-3486. [PubMed: 20392063]

18. Reddy, R.; Davis, B.; Carney, PS.; Bhargava, R. Modeling Fourier Transform Infrared Spectroscopic Imaging of Prostate and Breast Cancer Tissue Specimens. International Symposium on Biomedical Imaging; 2011. p. 738-741.

19. Davis BJ, Carney PS, Bhargava R. Theory of Mid-infrared Absorption Microspectroscopy: II. Heterogeneous Samples. Anal Chem. 2010; 82(9):3487-3499. [PubMed: 20392064]

20. Filik J, Frogley MD, Pijanka JK, Wehbe K, Cinque G. Electric Field Standing Wave Artifacts in FTIR Micro-spectroscopy of Biological Materials. Analyst. 2012; 137(4):853-861. [PubMed: 22231204]

21. Goodman, JW. Introduction to Fourier Optics. Englewood, CO: Roberts and Company Publishers; 2005.

22. Dirac, PAM. The Principles of Quantum Mechanics. Oxford, UK: Oxford University Press; 1958.

23. Levin IW, Bhargava R. Fourier Transform Infrared Vibrational Spectroscopic Imaging: Integrating Microscopy and Molecular Recognition. Annu Rev Phys Chem. 2005; 56:429-474. [PubMed: 15796707]

24. Stelzer EHK. Contrast, Resolution, Pixelation, Dynamic Range and Signal-To-Noise Ratio: Fundamental Limits to Resolution in Fluorescence Light Microscopy. Journal of Microscopy. 1998; 189(1):15-24.

25. Fernandez DC, Bhargava R, Hewitt SM, Levin IW. Infrared Spectroscopic Imaging for Histopathologic Recognition. Nat Biotechnol. 2005; 23(4):469-474. [PubMed: 15793574]

26. Bhargava R, Fernandez DC, Hewitt SM, Levin IW. High-Throughput Assessment of Cells and Tissues: Bayesian Classification of Spectral Metrics from Infrared Vibrational Spectroscopic Imaging Data. Biochim Biophys Acta. 2006; 1758(7):830-845. [PubMed: 16822477]

27. Bhargava R, Schwartz Perlman R, Fernandez DC, Levin IW, Bartick EG. Non-invasive Detection of Superimposed Latent Fingerprints and Inter-Ridge Trace Evidence by Infrared Spectroscopic Imaging. Anal Bio Chem. 2009; 394(8):2069-2075.

28. Bhargava R, Levin IW. Fourier transform infrared imaging: theory and practice. Anal Chem. 2001; 73(21):5157-5167. [PubMed: 11721913]

29. Griffiths, PR.; De Haseth, JA. Fourier Transform Infrared Spectroscopy. New York: John Wiley and Sons; 1986.

30. Bhargava R, Wang SQ, Koenig JL. Route to Higher-Fidelity FTIR Imaging. Appl Spectrosc. 2000; 54(4):486-495.

31. Reddy RK, Bhargava R. Accurate Histopathology from Low Signal-To-Noise Ratio Spectroscopic Imaging Data. Analyst. 2010; 135(11):2818-2825. [PubMed: 20830324]

32. Saleh, BEA.; Teich, MC. Fundamentals of Photonics. New York: John Wiley and Sons; 2007. 
33. Bhargava R. Infrared Spectroscopic Imaging: The Next Generation. Appl Spectrosc. 2012; 66(10): 1091-1120. [PubMed: 23031693]

\section{APPENDIX. DERIVATION OF THE TRANSFER FUNCTION OF THE SCHWARZSCHILD}

We model the Schwarzschild as a focusing system with two concentric apertures, one outer aperture with $\mathrm{NA}_{\text {out }}=\sin \left(\theta_{\text {out }}\right)$ and a second inner circular obstruction with $\mathrm{NA}_{\text {in }}=\sin \left(\theta_{\text {in }}\right)$ (see Fig. A1). The focal length, which is the distance between the Schwarzschild and the focal point, is assumed known.

Consider an object placed a distance $d_{1}$ from the Schwarzschild, as shown in Fig. A2. Let the field in a plane perpendicular to the principal axis at the object be $\left|U_{0}\right\rangle$. The field, $\left|U_{1}\right\rangle$, just before the Schwarzschild is given by:

$$
\left|U_{1}\right\rangle=\mathbf{K}_{d_{1}}\left|U_{0}\right\rangle
$$

The field immediately after the Schwarzschild, $\left|U_{2}\right\rangle$, can be expressed in terms of $\left|U_{1}\right\rangle$ and an operator, $\mathbf{G}_{C}$, corresponding to the transmission function of the Schwarzschild as:

$$
\begin{gathered}
\left|U_{2}\right\rangle=\mathbf{G}_{C}\left|U_{1}\right\rangle \\
\mathbf{G}_{C}=\int d^{2} r|\mathbf{r}\rangle Q_{C}(\mathbf{r}) \exp \left[i \frac{\pi \bar{\nu}}{L_{f}} r^{2}\right]\langle\mathbf{r}| \quad(\mathrm{A} 3)
\end{gathered}
$$

where $L_{f}$ is the focal length of the Schwarzschild. Note that the quadratic phase function in Eq. A3 is present because we have used a thin lens like approximation for the focusing system. $Q_{C}(\mathbf{r})=Q_{R_{1}}^{R_{2}}(\mathbf{r})$ is defined in Eq. A4, and $R_{1}$ and $R_{2}$ are the inner and outer aperture radii.

$$
Q_{a}^{b}(\mathbf{r})= \begin{cases}1 & a<r<b \\ 0 & \text { else }\end{cases}
$$

The field $\left|U_{3}\right\rangle$ in the image plane is

$$
\left|U_{3}\right\rangle=\mathbf{K}_{d_{2}}\left|U_{2}\right\rangle
$$

The term $f_{z}(\mathbf{f})$ in $\mathbf{K}_{d}$ can be approximated as $f_{z}(\mathbf{f}) \approx v\left(1-\left(f^{2} / 2 v^{\overline{2}}\right)\right)$. After substituting Eq. A2 and Eq. A1 in Eq. A5 and simplifying we obtain

$$
\begin{gathered}
\left|U_{3}\right\rangle=\mathbf{K}_{d_{2}} \mathbf{G}_{C} \mathbf{K}_{d_{1}}\left|U_{0}\right\rangle \\
=\int d^{2} r d^{2} r^{\prime} d^{2} r^{\prime \prime}|\mathbf{r}\rangle Q_{R_{1}}^{R_{2}}\left(\mathbf{r}^{\prime}\right)\left\langle\mathbf{r}^{\prime} \mid U_{0}\right\rangle \exp \left[i \frac{2 \pi \bar{\nu}}{d_{1}} \mathbf{r} \cdot \mathbf{r}^{\prime}\right] \\
\times \exp \left[i \frac{2 \pi \bar{\nu}}{d_{2}} \mathbf{r}^{\prime \prime} \cdot \mathbf{r}\right] \exp \left[i \frac{2 \pi \bar{\nu}}{2 d_{2}} r^{\prime \prime 2}\right] \exp \left[i \frac{2 \pi \bar{\nu}}{2 d_{1}} r^{\prime 2}\right] \\
\times \exp \left[i \frac{2 \pi \bar{\nu}}{2}\left(\frac{1}{d_{1}}+\frac{1}{d_{2}}-\frac{1}{L_{f}}\right) r^{2}\right]
\end{gathered}
$$

This is the relation between the image and the object for a Schwarzschild. In most FT-IR imaging systems, we can make a few approximations and simplify Eq. 26. The object size 
(illuminated area of the sample) is typically much smaller than the size of the

Schwarzschild. This means that $\exp \left[i\left(2 \pi v / 2 d_{1}\right) r^{\prime 2}\right] \approx 1$. The image size is also much smaller than the Schwarzschild dimensions; therefore, $\exp \left[i\left(2 \pi v / 2 d_{2}\right) r^{\prime \prime 2}\right] \approx 1$. We know that ${ }^{32}$ for a thin lens (or, more generally, for a system that focuses light like a thin lens), the position of the image and object are related by $\left(1 / d_{1}\right)+\left(1 / d_{2}\right)=\left(1 / L_{f}\right)$. Therefore, $\exp \left\{i(2 \pi v / 2)\left[\left(1 / d_{1}\right)+\right.\right.$ $\left.\left.\left(1 / d_{2}\right)-\left(1 / L_{f}\right)\right]\left(x^{2}+y^{2}\right)\right\}=1$.

With these approximations, Eq. A6 reduces to:

$$
\left|U_{3}\right\rangle=\int d^{2} r d^{2} r^{\prime} d^{2} r^{\prime \prime}|\mathbf{r}\rangle \mathrm{Q}_{R_{1}}^{R_{2}}\left(\mathbf{r}^{\prime}\right)\left\langle\mathbf{r}^{\prime} \mid U_{0}\right\rangle \times \exp \left[i \frac{2 \pi \bar{\nu}}{d_{1}} \mathbf{r} \cdot \mathbf{r}^{\prime}\right] \exp \left[i \frac{2 \pi \bar{\nu}}{d_{2}} \mathbf{r}^{\prime \prime} \cdot \mathbf{r}\right]
$$

Defining magnification $M_{C}=\left(d_{2} / d_{1}\right)$ and evaluating this integral after projecting on the Fourier basis yields

$$
\left\langle\mathbf{f} \mid U_{3}\right\rangle=Q_{R_{1}}^{R_{2}}\left(-\frac{\mathbf{f} d_{2}}{\bar{\nu}}\right)\left\langle-M_{C} \mathbf{f} \mid U_{0}\right\rangle
$$

It can be noted that the system is linear, but not shift invariant (because of the magnification term). With this caveat, we can think of $Q_{R_{1}}^{R_{2}}\left(-\mathbf{f} d_{2} / \bar{\nu}\right)$ as the Schwarzschild transfer function. In our model, the exact values of focal lengths are not required, provided that the system is in focus. Only the outer and inner numerical apertures of the Schwarzschilds and the magnification factors are required.

For a Schwarzschild with an orientation as in Fig. 8, typically the image is close to the focus. Thus, we can make approximations that $d_{2} \approx d_{2} \approx L_{f}$ ( $L_{f}$ is focal length), $\mathrm{NA}_{\text {out }} \approx$ $\left(R_{2} / d_{2}\right)$ and $\mathrm{NA}_{\text {in }} \approx\left(R_{1} / d_{1}\right)$. Also note that $\mathbf{Q}$ is an even function. This gives

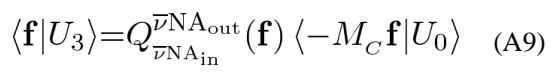

Note that propagation in free space is a spatial frequency band pass $Q_{0}^{\bar{\nu}}(\mathbf{f})$ and spatial frequencies beyond $v$ do not reach the detector. For a Schwarzschild with an orientation as in Fig. A9, the object is close to the focus and so we can make the approximations that $d_{1} \approx$ $L_{f}, \mathrm{NA}_{\text {out }} \approx\left(R_{2} / d_{1}\right)$ and $\mathrm{NA}_{\mathrm{in}} \approx\left(R_{1} / d_{1}\right)$. This gives

$$
\begin{aligned}
& \left\langle\mathbf{f} \mid U_{3}\right\rangle=Q_{\overline{\nu N A_{\text {in }}}}^{\bar{\nu} \mathrm{N}_{\text {out }}}\left(M_{C} \mathbf{f}\right)\left\langle-M_{C} \mathbf{f} \mid U_{0}\right\rangle \\
& \left\langle-\mathbf{f} / M_{C} \mid U_{3}\right\rangle=Q_{\bar{\nu} \mathrm{NA}_{\text {in }}}^{\overline{\nu N A_{\text {out }}}}(\mathbf{f})\left\langle\mathbf{f} \mid U_{0}\right\rangle \quad \text { (A11) }
\end{aligned}
$$

\section{Point-Spread Functions and Resolution}

Point-spread functions for the two configurations in the article Table I are presented in Fig. A4.

Simulation data for two point objects separated by four different distances are presented in Figs. A5 and A6. Data in Fig. A5 correspond to the high-NA configuration in the article Table I, and Fig. A6 corresponds to the lower-NA configuration. All data are at $3950 \mathrm{~cm}^{-1}$. 
Axes in all the images are scaled to the effective size of the detector-intensity-image at the sample. From these images, it is evident that two points separated by $1 \mu \mathrm{m}$ cannot be resolved with either $\mathrm{NA}_{C_{2} \text { out }}=0.65$ or $\mathrm{NA}_{C_{2} \text { out }}=0.5$, whereas they can be resolved in both configurations at a separation of $4 \mu \mathrm{m}$. However, $\mathrm{NA}_{C_{2} \text { out }}=0.65$ can resolve two points separated by $2.4 \mu \mathrm{m}$ (which is the resolution according to Rayleigh criterion), while $\mathrm{NA}_{C_{2} \text { out }}$ 0.5 cannot. Points at a separation of $3 \mu \mathrm{m}$ can be just resolved with $\mathrm{NA}_{C_{2} \text { out }}=0.5$. Data presented here illustrate the best image quality obtainable from systems with the optical configurations in the article Table I, without discretization (i.e., using an analog detector). Discretized data obtained at the optimal pixel size (as calculated in section "Pixel Size" in the article) have all the information needed to construct images of this quality.

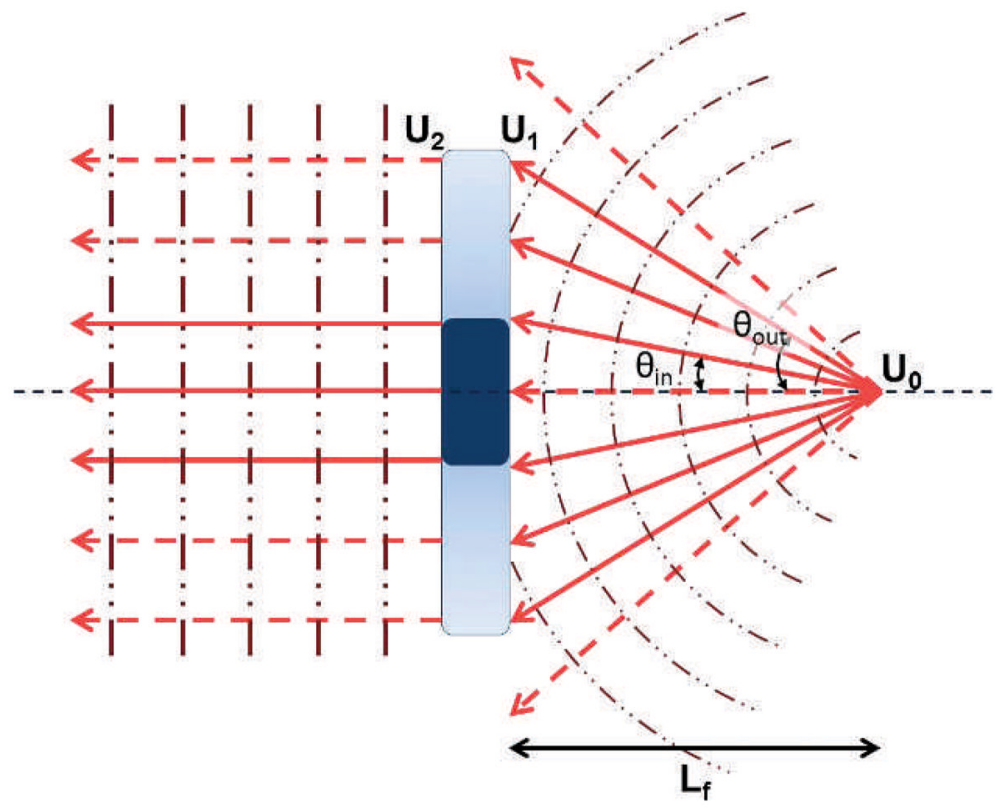

Fig. A1.

A Schwarzschild model. A point source placed at the focus results in a plane wave. The outer and inner numerical apertures limit the angles that enter the Schwarzschild.

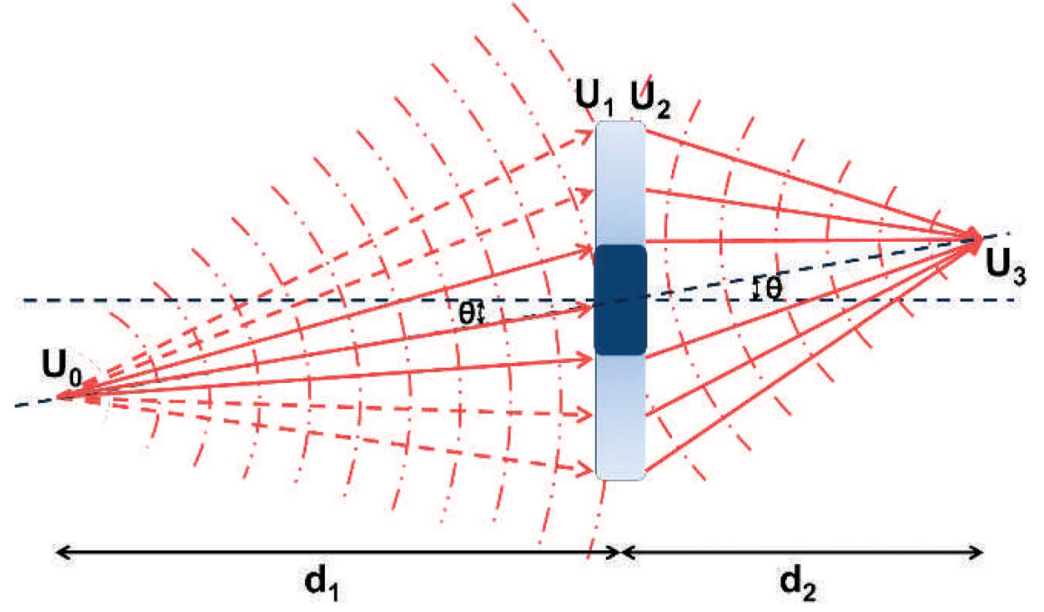

Fig. A2. 
An object placed at distance $d_{1}$ from the Schwarzschild produces an image at position $d_{2}$ on the other side.

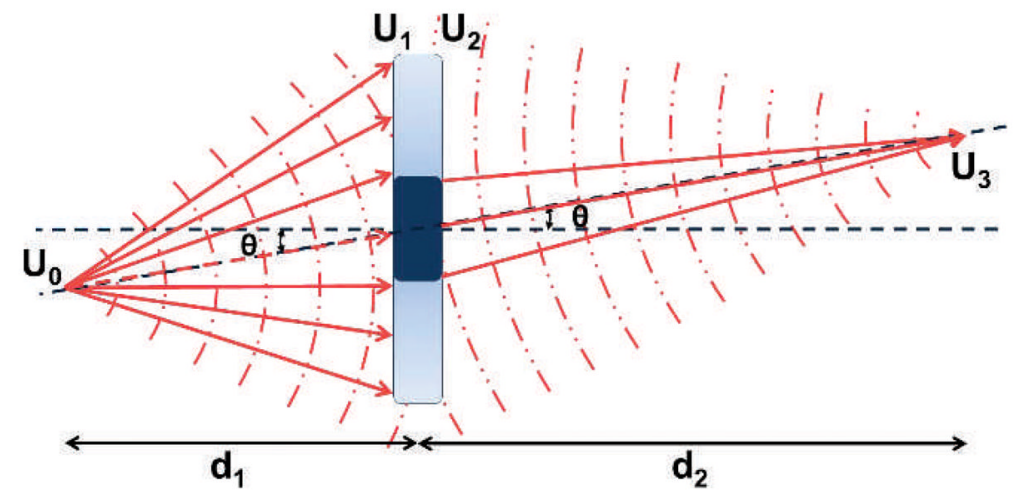

Fig. A3.

The Schwarzschild is flipped, and the object distance $d_{1}$ is smaller than the image distance $d_{2}$ on the other side. Equivalently, a larger range of angles are accepted by the Schwarzschild from the object side.
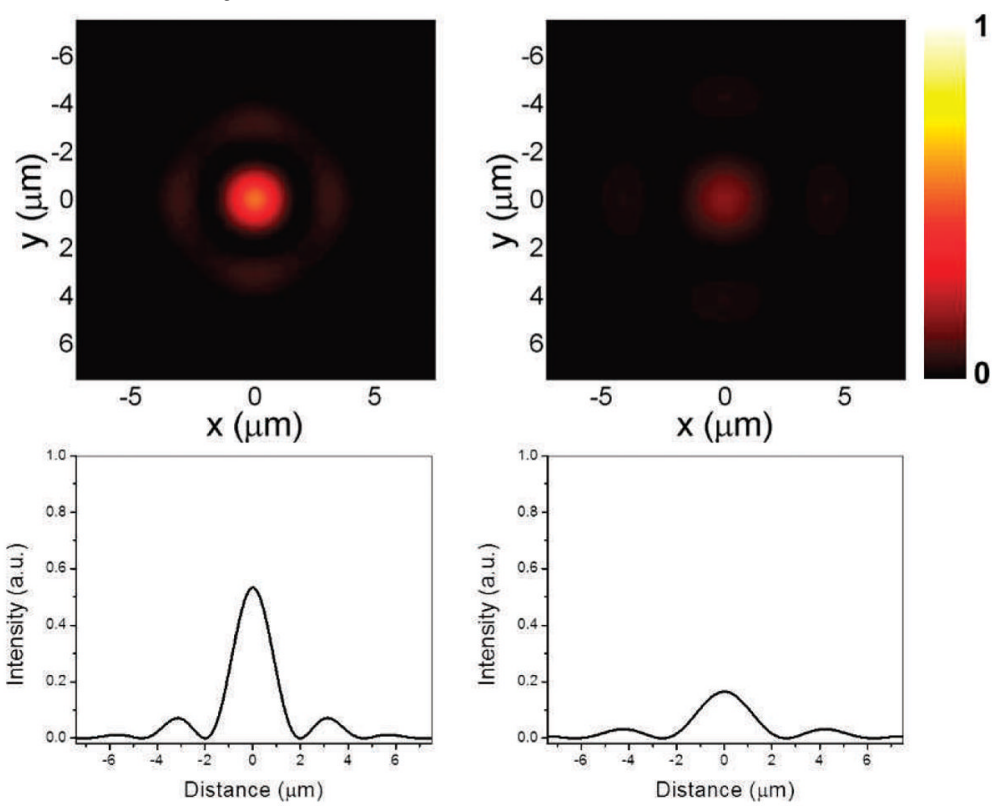

Fig. A4.

Point-spread functions. Data on the left correspond to a configuration with $\mathrm{NA}_{C_{2} \text { out }}=0.65$ and data on the right corresponds to a configuration with $\mathrm{NA}_{C_{2} \text { out }}=0.5$. 

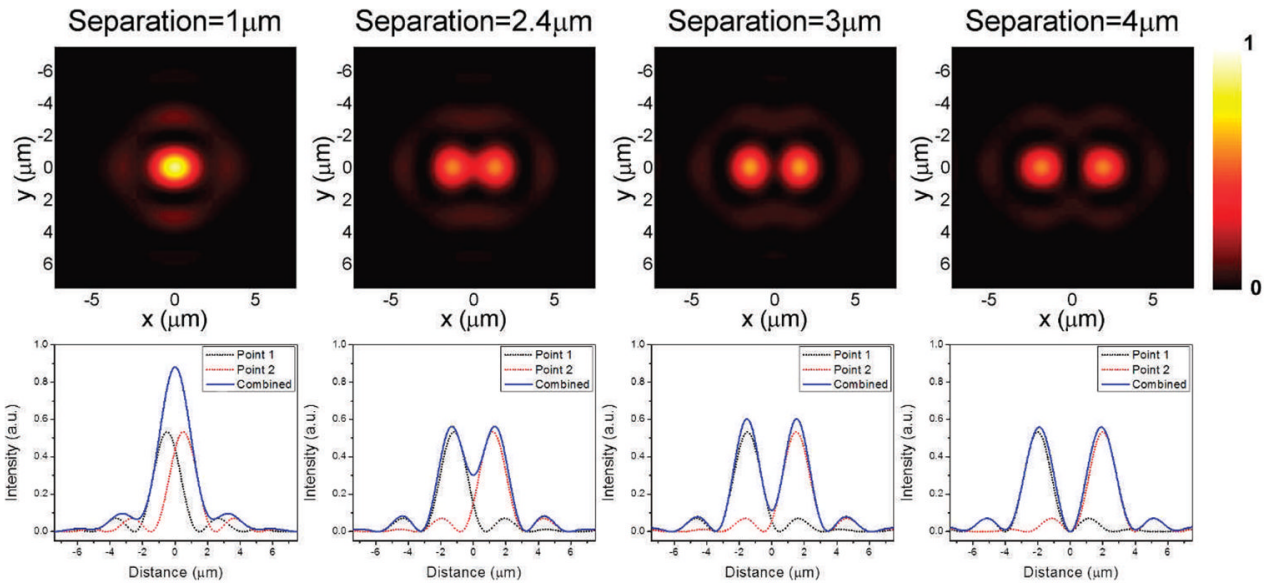

Fig. A5.

Intensity at the detector plane at $3950 \mathrm{~cm}^{-1}$ for two point objects separated by distances (indicated on top). Corresponding profile plots at $y=0$ are presented in the bottom row. Schwarzschild $C_{2}$ has $\mathrm{NA}_{C_{2} \text { out }} 0.65$. Axes are scaled to the effective image size at the sample.
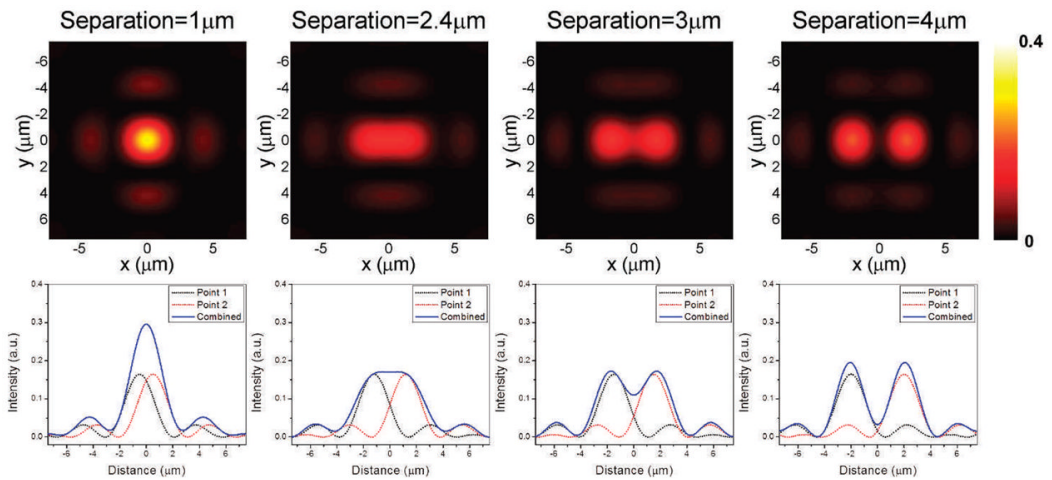

Fig. A6.

Intensity at the detector plane at $3950 \mathrm{~cm}^{-1}$ for two point objects separated by distances (indicated on top). Corresponding profile plots at $y=0$ are presented in the bottom row. Schwarzschild $C_{2}$ has $\mathrm{NA}_{C_{2} \text { out }}=0.5$. Axes are scaled to the effective image size at the sample. 


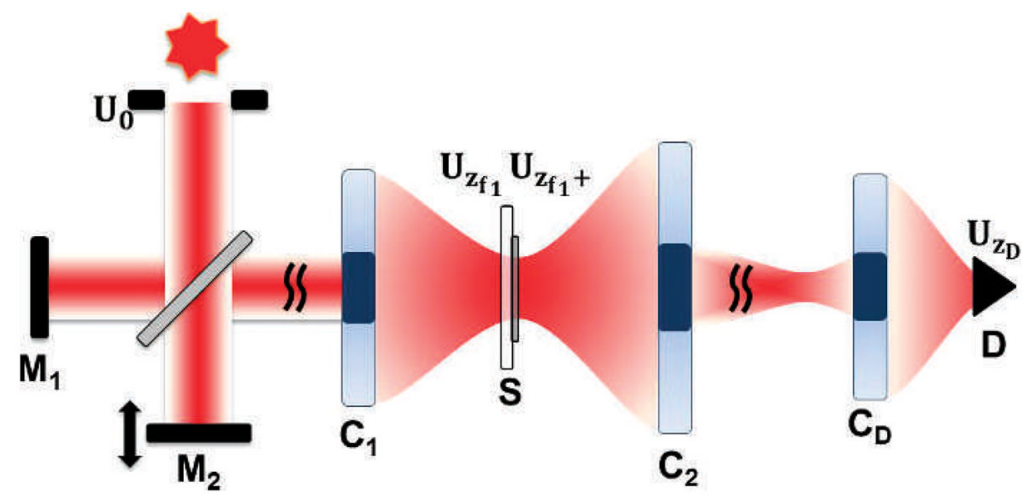

Fig. 1.

Model for a FT-IR imaging system. The system consists of an interferometer coupled to a microscope system. Schwarzschild $C_{1}$ focus light on to the sample $S, C_{2}$, and $C_{\mathrm{D}}$ transmit light from the sample to the detector $D$. The fields in different planes are also indicated. 

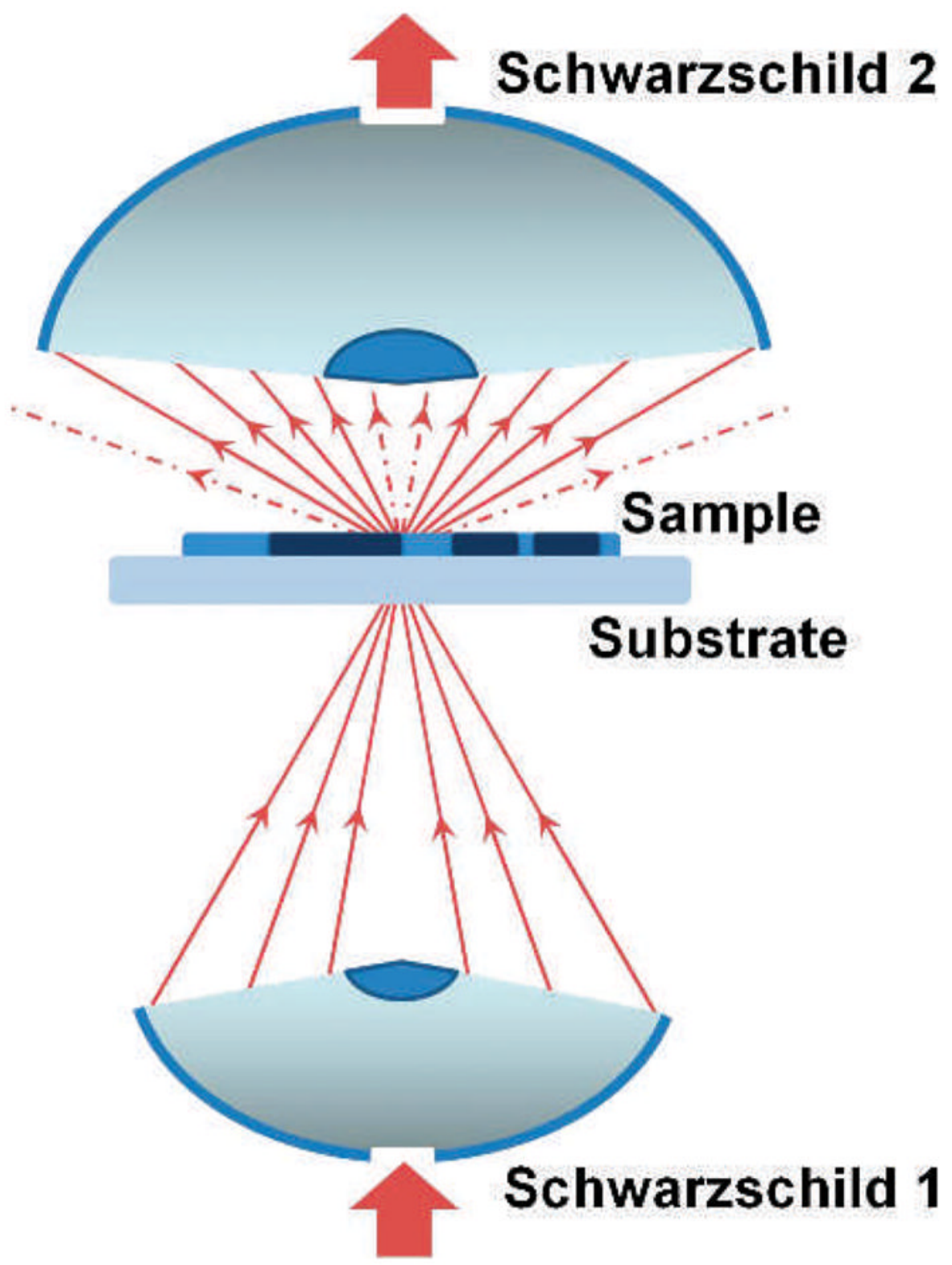

Fig. 2.

Schwarzschild $C_{1}$, which focuses light on to the sample, and $C_{2}$, the collection Schwarzschild, are shown. 

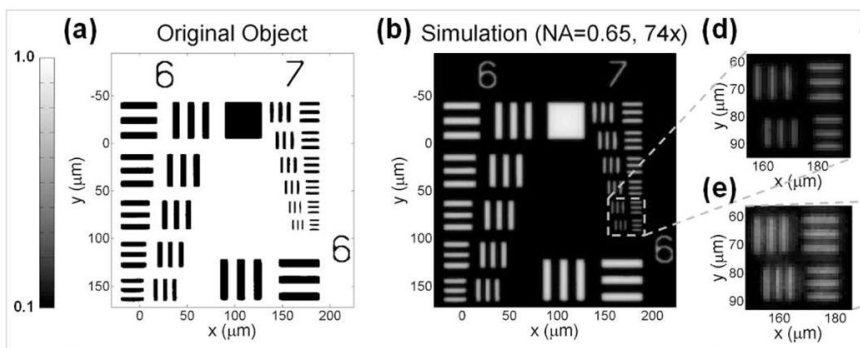

(c) Recorded (NA=0.65, 74x)

(f) Simulation (NA=0.5, 15x)

(g) Simulation $(\mathrm{NA}=0.5,15 \mathrm{x})$ (i)
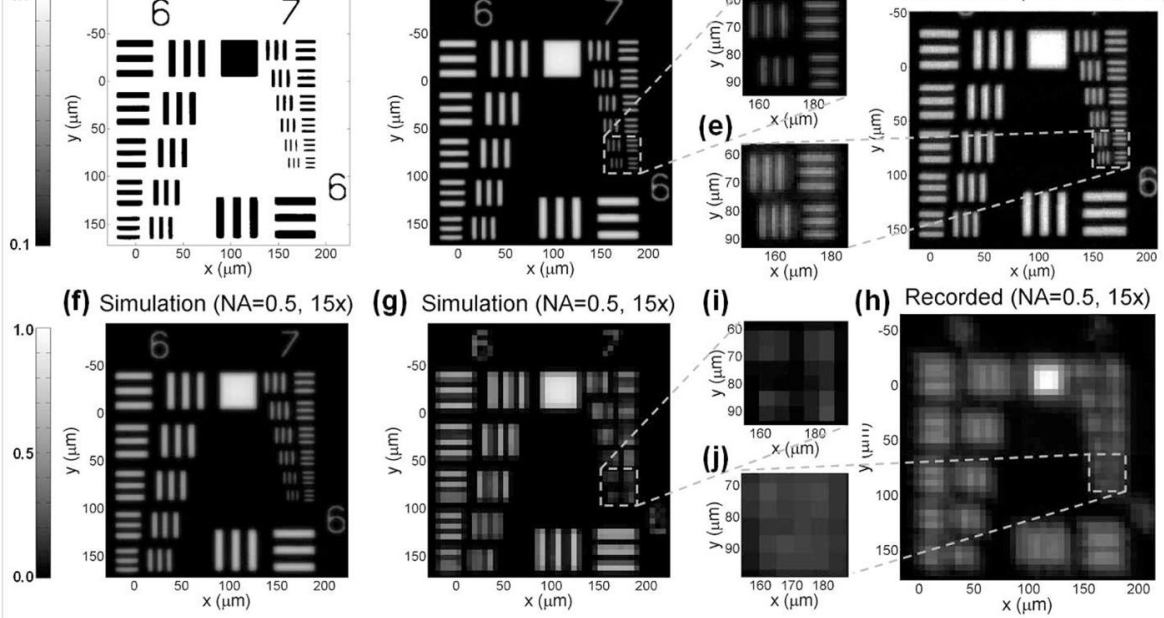

(h) Recorded (NA=0.5, 15x)

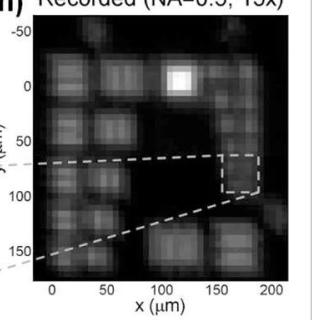

Fig. 3.

(a) The original object consists of a set of bars of different sizes with transmittance indicated to the left. Absorbance images from simulations at $v=3850 \mathrm{~cm}^{-1}$ are shown when Schwarzschild $C_{2}$ has (b) NA $=0.65$, (f) NA $=0.50,(\mathrm{~g}) \mathrm{NA}=0.50$. The pixel size in (b) and (f) are $1.1 \mu \mathrm{m}$, whereas the pixel size in (g) is $5.5 \mu \mathrm{m}$. Experimental absorbance images from $\nu=3950 \mathrm{~cm}^{-1}$ are shown with configurations (c) NA $=0.65$, pixel size $=1.1 \mu \mathrm{m}$; (h) NA = 0.50 , pixel size $=5.5 \mu \mathrm{m}$; (d), (e), (i), (j) magnified regions from corresponding images . 
(a)

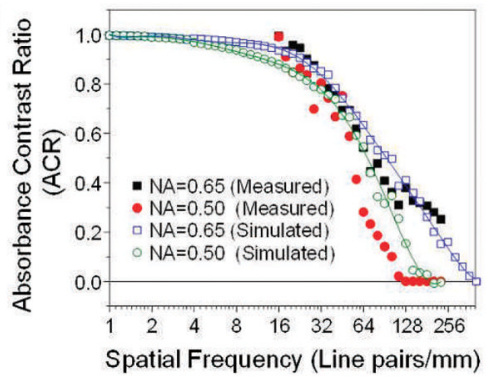

(b)

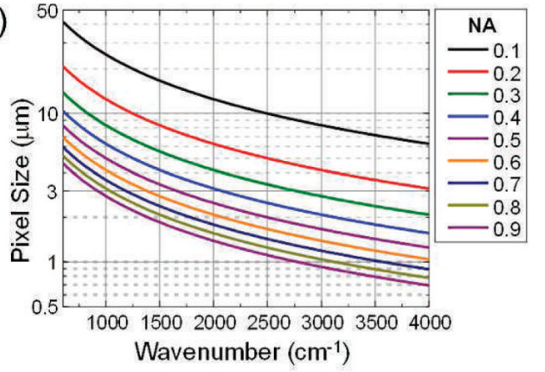

Fig. 4.

(a) Plot of absorbance contrast ratio (ACR) measured from both simulation and recorded experimental data as a function of spatial frequency at $v=3850 \mathrm{~cm}^{-1}$ for the two systems in Table I. (b) Plot of minimum pixel size required for correctly sampling allowable spatial frequencies as a function of $v$ and NA is shown. The pixel size corresponds to the effective pixel size at the sample. 


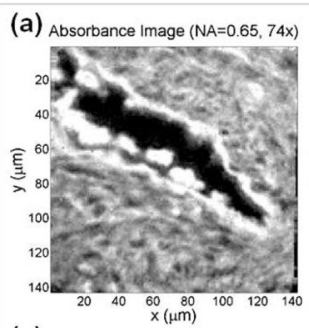

(e)

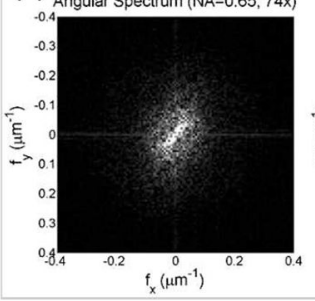

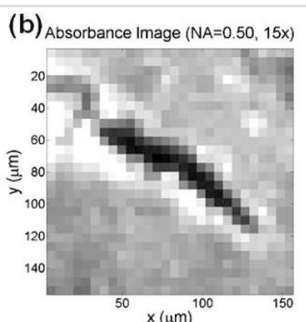

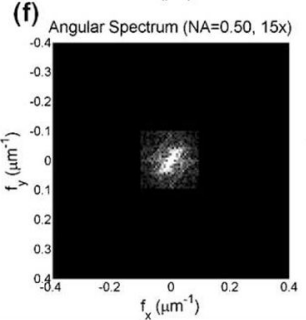

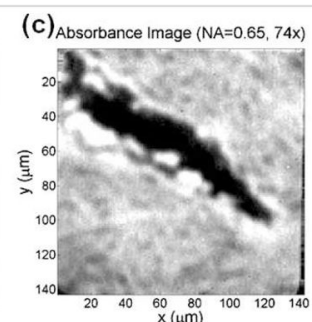

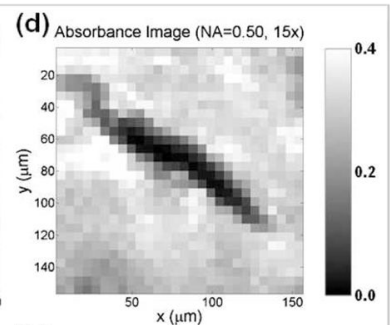

(h)
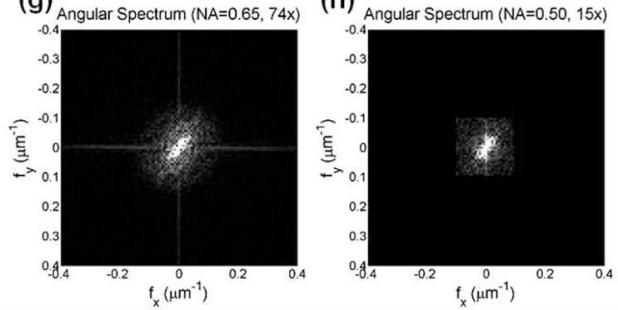

\section{Fig. 5.}

Absorbance images from spectroscopic imaging data obtained from the two configurations $(\mathrm{NA}=0.65, \mathrm{NA}=0.50)$ are shown on top. The corresponding angular spectra are shown at the bottom. Images for the system with (a) NA $=0.65$ and (b) NA $=0.50$ obtained by plotting the absorbance at $v=2962 \mathrm{~cm}^{-1}$. Similarly, the absorbance at $v=1650 \mathrm{~cm}^{-1}$ is shown for systems with (c) NA $=0.65$ data and (d) NA $=0.50$. 

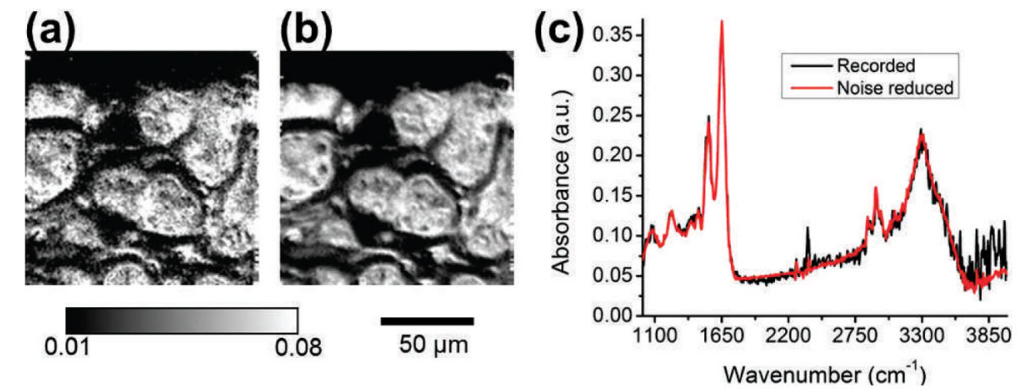

Fig. 6.

Infrared spectroscopic imaging data from breast tissue before and after noise reduction. (a) An absorbance image at the asymmetric $\mathrm{C}-\mathrm{H}$ stretching mode before noise reduction and the corresponding image (b) after noise reduction. (c) Spectra corresponding to recorded data and data after noise reduction from the same pixel. 
TABLE I

Experimental parameters used for data acquisition and modeling.

\begin{tabular}{lll}
\hline Parameter & System 1 & System 2 \\
\hline $\mathrm{NA}_{C_{1} \text { out }}$ & 0.5 & 0.5 \\
$\mathrm{NA}_{C_{2} \text { out }}$ & 0.5 & 0.65 \\
$\mathrm{NA}_{C_{D^{\text {out }}}}$ & 0.5 & 0.5 \\
$M_{C_{1}}$ & $15 \times$ & $15 \times$ \\
$M_{C_{2}}$ & $15 \times$ & $74 \times$ \\
$M_{C_{\mathrm{D}}}$ & $3 \times$ & $3 \times$ \\
Pixel size (effective) & $5.5 \mu \mathrm{m}$ & $1.115 \mu \mathrm{m}$ \\
Spectral resolution & $8 \mathrm{~cm}-1$ & $8 \mathrm{~cm}^{-1}$ \\
No. of scans per pixel & 8 & 128 \\
No. of scans (background) & 128 & 128 \\
No. of detector elements & $128 \times 128$ & $128 \times 128$ \\
Undersampling ratio & 2 & 2 \\
\hline
\end{tabular}


TABLE II

A list of symbols used along with their description.

\begin{tabular}{|c|c|}
\hline Symbol & Description \\
\hline$v^{-}$ & Wavenumber \\
\hline $\mathrm{NA}_{C_{i} \text { out }}, \mathrm{NA}_{C_{i} \text { in }}$ & Outer and inner numerical aperture of Schwarzschild $C_{i}$ \\
\hline $\mathbf{r}=(x, y), \mathbf{r}^{\prime} \mathbf{r}^{\prime \prime}$ & Coordinate space variables \\
\hline $\mathbf{f}=\left(f_{x} f_{y}\right), \mathbf{f}_{1}, \mathbf{f}_{2}$ & Transverse spatial frequency variables \\
\hline$f_{z}$ & Longitudinal spatial frequency variable \\
\hline$z$ & Axial position (along the principal axis of light propagation) \\
\hline$z_{\mathrm{D}}$ & Axial detector position \\
\hline$z_{\mathrm{I}}$ & Beam splitter position \\
\hline$z_{C_{i}}$ & Axial position of Schwarzschild $C_{1}$ \\
\hline$z_{f_{i}}$ & Axial position where light is focused by Schwarzschild $C_{1}$ \\
\hline$d_{1}, d_{2}$ & Light propagation distances \\
\hline$d_{A_{1}}, d_{A_{2}}$ & Light propagation distances along each arm of the interferometer \\
\hline$L_{f_{i}}$ & Focal length of Schwarzschild $C_{1}$ \\
\hline$L_{\text {pixel }}$ & Length of (one side of) a pixel \\
\hline$A_{0}, B_{0}$ & Constants \\
\hline $\mathrm{U}(\mathbf{r}), \mathrm{U}^{*}(\mathbf{r})$ & Field in any plane and its corresponding complex conjugate respectively \\
\hline$\tilde{U}(\mathbf{f})$ & Fourier transform of the field in a plane \\
\hline$\delta(\mathbf{f})$ & Two-dimensional Dirac delta function \\
\hline$I_{0}(\mathbf{r})$ & Source intensity \\
\hline$I(\mathbf{r})$ & Intensity in a plane \\
\hline$h\left(\mathbf{r} ; \mathbf{r}^{\prime}\right)$ & Transfer function for coherent light \\
\hline$\left|U_{z_{i}}\right\rangle$ & Field in a plane at $z=z_{1}$ \\
\hline$\left\langle U_{z_{i}}\right|$ & Hermitian adjoint of field in a plane at $z=z_{1}$ \\
\hline$\left|U_{z f_{i}+}\right\rangle$ & Field immediately after the plane at $z=z_{f_{i}}$ \\
\hline$\left|U_{0}\right\rangle$ & Field from the source \\
\hline$\left|U_{i}\right\rangle$ & Field in plane $i$, for $I=1,2,3$ \\
\hline$|S\rangle$ & Sample \\
\hline$Q_{C_{i}}$ & Aperture function of Schwarzschild $C_{1}$ \\
\hline$M_{C_{i}}$ & Magnification of Schwarzschild $C_{1}$ \\
\hline$M=M_{C_{D}} M_{C_{2}}$ & Net magnification between the sample and the detector \\
\hline $\mathbf{A}$ & Operator for the entire optical imaging system \\
\hline $\mathbf{K}_{d}$ & Propagation operator for a propagation distance $d$ \\
\hline $\mathbf{I}_{d_{\mathrm{A}_{1}}, d_{\mathrm{A}_{2}}}$ & Operator for the interferometer where $d_{\mathrm{A}_{1}}, d_{\mathrm{A}_{2}}$ are path lengths along the arms \\
\hline $\mathbf{H}_{C_{i}}$ & Transfer function (operator) for a Schwarzschild $C_{1}$ in focus \\
\hline $\mathbf{G}_{C_{i}}$ & Transmission function (operator) of Schwarzschild $C_{1}$ \\
\hline
\end{tabular}

